\title{
A Global Attractor in Some Discrete Contest Competition Models with Delay under the Effect of Periodic Stocking
}

\author{
Ziyad AlSharawi \\ Department of Mathematics and Statistics, Sultan Qaboos University, P.O. Box 36 123, Al-Khod, Oman \\ Correspondence should be addressed to Ziyad AlSharawi; alshalzm@alsharawi.info
}

Received 17 June 2013; Revised 3 September 2013; Accepted 16 September 2013

Academic Editor: Yanni Xiao

Copyright (C) 2013 Ziyad AlSharawi. This is an open access article distributed under the Creative Commons Attribution License, which permits unrestricted use, distribution, and reproduction in any medium, provided the original work is properly cited.

\begin{abstract}
We consider discrete models of the form $x_{n+1}=x_{n} f\left(x_{n-1}\right)+h_{n}$, where $h_{n}$ is a nonnegative $p$-periodic sequence representing stocking in the population, and investigate their dynamics. Under certain conditions on the recruitment function $f(x)$, we give a compact invariant region and use Brouwer fixed point theorem to prove the existence of a $p$-periodic solution. Also, we prove the global attractivity of the $p$-periodic solution when $p=2$. In particular, this study gives theoretical results attesting to the belief that stocking (whether it is constant or periodic) preserves the global attractivity of the periodic solution in contest competition models with short delay. Finally, as an illustrative example, we discuss Pielou's model with periodic stocking.
\end{abstract}

\section{Introduction}

In mathematical ecology, difference equations of the form $x_{n+1}=x_{n} f\left(x_{n}\right), n \in \mathbb{N}:=\{0,1, \ldots\}$ are used to model single species with nonoverlapping generations $[1,2]$, where $x_{n}$ denotes the number of sexually mature individuals at discrete time $n$, and $f\left(x_{n}\right)$ is the density-dependent net growth rate of the population. The form of the function $f(x)$ is chosen to reflect certain characteristics of the studied population such as intraspecific competition. For some background readings about models obtained by the various choices of $f(x)$, we refer the reader to $[1,3,4]$ in the discrete case. Also, we refer the reader to [5] and the references therein for the continuous case. Two classical types are known as the scrambled and contest competition models [4]. Our attention in this work is limited to the contest competition models where $f(x)$ is assumed to be decreasing, $x f(x)$ is increasing, and $x f(x)$ is asymptotic to a certain level at high population densities. A prototype of such models is the Beverton-Holt model [6], which is obtained by considering $f(x)=\mu K x /(K+(\mu-$ 1) $x$ ). Here, $\mu>1$ is interpreted as the growth rate per generation, and $K$ is the carrying capacity of the environment. In populations with substantial time needed to reach sexual maturity, certain delay effect must be included in the function $f(x)$, which motivates us to consider difference equations of the form

$$
x_{n+1}=x_{n} f\left(x_{n-k}\right),
$$

where $k$ is a fixed positive integer [7]. In general, it is widely known that long time delay has a destabilizing effect on the population's steady state, while short time delay can preserve stability [8-10]. However, when the delay is large, the dynamics of (1) is less tractable [11]. Furthermore, we are more interested here in the effect of stocking than the effect of delay, and therefore, we keep the time delay short to preserve stability in the absence of stocking. In particular, we fix the delay to be $k=1$.

A substantial body of research has explored the effect of constant stocking on population models without delay [1219]. In brief and general terms, it has been found that constant stocking can be used to suppress chaos, reverse the period doubling phenomena, lower the risk of extinction, and have a stabilizing effect on the population steady state. On the other hand, and to the best of our knowledge, little (if any) has been done to explore the effect of stocking (whether constant 
or periodic) on models with delay. So, our work here has a twofold objective which motivates us to consider difference equations of the form to study the effect of periodic stocking on contest competition models with delay and to complement the work of the author and his collaborators in [20], where the dynamics of (1) with $k=1$ was studied under the effect of constant yield harvesting. Recall that we have some accumulating restrictions on the function $f(x)$ due to the nature of associating our equation with contest competition models. So, in an abstract mathematical form, our problem can be posed as follows. Consider the difference equation

$$
x_{n+1}=x_{n} f\left(x_{n-1}\right)+h_{n}
$$

where $\left\{h_{n}\right\}$ is a nonnegative $p$-periodic sequence representing stocking due to refuge, immigration, feeding, and so forth, and the function $f(x)$ obeys the following conditions:

(C1) $f(0)=b>1$,

(C2) $f \in C^{1}([0, \infty))$ and $f(x)$ is decreasing on $[0, \infty)$,

(C3) $x f(x)$ is increasing and bounded.

The condition in (C1) is a generic one in the absence of stocking, that is, if $b \leq 1$ and $h_{n}=0$, then there is no long-term survival regardless of the initial density of the population.

This paper is organized as follows. In Section 2, we give some preliminary results concerning local stability, boundedness, and global stability of (2) when the stocking sequence is 1 -periodic, that is, when $h_{n}=h>0$ for all $n \in \mathbb{N}$. In Section 3, the period of the stocking sequence is taken to be larger than one. A compact invariant region has been established and a characterization of the periodic solutions is given. Also, the global asymptotic behavior of solutions has been investigated when $p=2$. As a particular case of (2), we discuss Pielou's equation with delay one in Section 4.

\section{Preliminary Results: The Autonomous Case}

In this section, we focus on the autonomous case, that is, $h_{j}=$ $h>0$ for all $j=0,1, \ldots, p-1$. Thus, (2) becomes

$$
x_{n+1}=x_{n} f\left(x_{n-1}\right)+h .
$$

Some results concerning (3) can be found in the literature [21]; however, for the sake of completeness and usage in the nonautonomous case, we give the following preliminary results.

2.1. Local Stability and Boundedness. Equation (3) has two equilibrium solutions at $h=0$, namely, 0 and $f^{-1}(1)$. For $h>0$, the origin slides downward to become negative, while the other equilibrium stays positive and slides upward. This fact becomes clear when we write $t=t f(t)+h$ as $1-h / t=f(t)$. The left hand side is increasing in $t$ while the right hand side is decreasing. Thus, we have only one positive equilibrium in the positive quadrant, which we denote in the sequel by $\bar{x}_{2, h}$. Since $\bar{x}_{2, h}$ is positive and increasing in $h, f\left(\bar{x}_{2, h}\right)<1$ for all $h>0$. The linearized equation associated with (3) at a fixed point $\bar{x}$ is given by

$$
y_{n+1}-f(\bar{x}) y_{n}-\bar{x} f^{\prime}(\bar{x}) y_{n-1}=0
$$

Define $p:=f(\bar{x})$ and $q:=-\bar{x} f^{\prime}(\bar{x})$. For $\bar{x}=\bar{x}_{2, h}$, we have $0<p<1$ and $q$ is nonnegative. The roots of $\lambda^{2}-p \lambda+q=0$ determine the local stability of our equilibrium point. Since $\lambda_{j, h}=(1 / 2)\left(p+(-1)^{j} \sqrt{p^{2}-4 q}\right), j=1,2, \bar{x}_{2, h}$ starts as stable at $h=0$ and stays stable as long as $q<1$. Figure 1 clarifies the relationship between $p, q$ and the magnitude of $\lambda_{j, h}$. We summarize these facts in the following proposition.

Proposition 1. Assume that conditions (C1) to (C3) are satisfied and $h>0$. The positive equilibrium $\bar{x}_{2, h}$ of (3) is locally asymptotically stable.

Proof. Since $\bar{x}_{2, h}>\bar{x}_{2,0}$, we have $p<1$. Also, since $F(t)=$ $t f(t)$ is increasing, we obtain $F^{\prime}(t)=t f^{\prime}(t)+f(t)>0$ and consequently $-q+p>0$. Thus, we have $q<p<1$. Now, Figure 1 makes the rest of the proof clear.

It is obvious that $x_{k} \geq h$ for all $k \geq 1$. On the other hand, since

$$
\begin{aligned}
x_{n+1}= & x_{n-1} f\left(x_{n-1}\right) f\left(x_{n-2}\right)+h f\left(x_{n-1}\right)+h \\
& \leq x_{n-1} f\left(x_{n-1}\right) b+h b+h,
\end{aligned}
$$

the boundedness of $y=t f(t)$ assures the boundedness of all solutions of (2).

2.2. Oscillations and Global Stability. A solution of (3) is called oscillatory if it is neither, eventually, less than nor larger than $\bar{x}_{2, h}$ [11]. Also, one can consider oscillations about a curve [20]. A solution $\left\{x_{n}\right\}$ of (3) is called oscillatory about a curve $H(x, y)=0$ if the sequence $\left\{u_{n}=\left(x_{n-1}, x_{n}\right)\right\}$ does not eventually stay on one side of the curve. The latter definition can be more convenient in some cases; however, in (3), both are equivalent when we consider $H(x, y)=y-x$ as we show in the following result.

Proposition 2. A solution of (3) is oscillatory if and only if it is oscillatory about the curve $y=x$.

Proof. Assume that $\left\{x_{n}\right\}$ oscillates about $\bar{x}_{2, h}$, but it is not oscillatory about $y=x$. So, $\left\{x_{n}\right\}$ is either eventually increasing or eventually decreasing, which contradicts the assumption that $x_{n}$ is oscillatory about $\bar{x}_{2, h}$. Conversely, suppose $\left\{\left(x_{n-1}, x_{n}\right)\right\}$ oscillates about $y=x$, but $\left\{x_{n}\right\}$ does not oscillate about $\bar{x}_{2, h}$. First, we consider the case $x_{n} \leq \bar{x}_{2, h}$ for all $n \geq n_{0}$. If $x_{m}>x_{m-1}$ for some $m>n_{0}$, then $f\left(x_{m}\right)<f\left(x_{m-1}\right)$ and consequently

$$
x_{m+1}=x_{m} f\left(x_{m-1}\right)+h>x_{m} f\left(x_{m}\right)+h>x_{m} .
$$

So, we can induce an eventually increasing sequence which contradicts our assumption. If $x_{m} \leq x_{m-1}$ for some $m>n_{0}$, then $x_{m+1} \leq x_{m} f\left(x_{m}\right)+h$. Thus, either $x_{m+1} \leq x_{m}$, and the induction leads to a decreasing sequence that must 


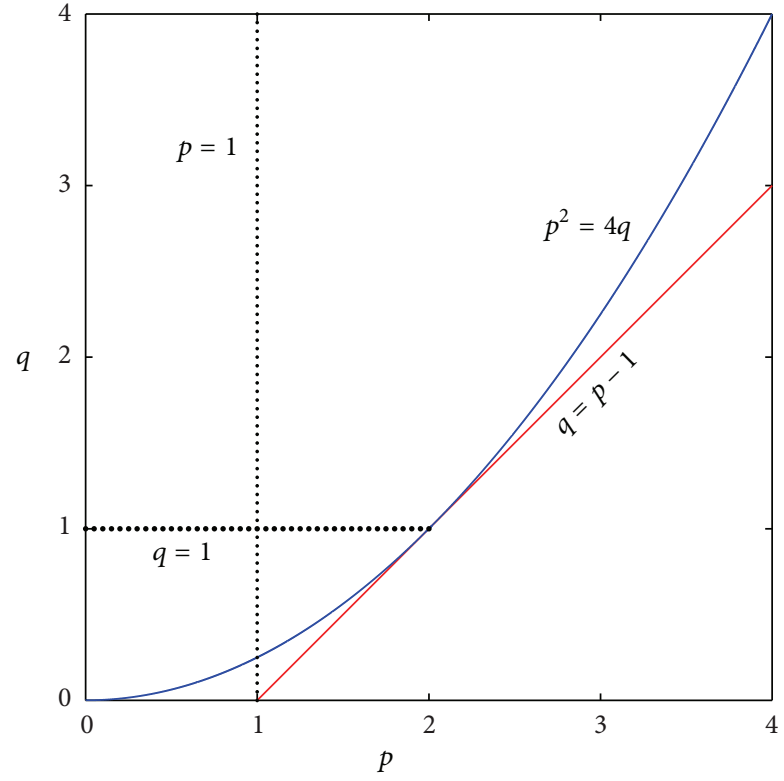

FIGURE 1: This figure shows the magnitude of the characteristic roots of (4) depending on the location of the $(p, q)$ values, where $p=f(\bar{x})$ and $q=-\bar{x} f^{\prime}(\bar{x})$. Also, $\left|\lambda_{1, h}\right|>1$ and $\left|\lambda_{1, h}\right|>1$ when $p, q>1 ;\left|\lambda_{1, h}\right|<1$ and $\left|\lambda_{1, h}\right|<1$ when $p<1$ and $q<1$.

converge which is not possible, or $x_{m+1}>x_{m}$, and we go back to the first scenario. Finally, the case $x_{n} \geq \bar{x}_{2, h}$ for all $n \geq n_{0}$ can be handled similarly.

Next, we define the map

$$
T(x, y)=(y, y f(x)+h) .
$$

The map $T$ portrays the solutions of (3) geometrically in the nonnegative quadrant, and therefore, it plays a prominent role in the sequel. Here, we used the nonnegative quadrant to denote the positive quadrant union of the axes on the boundary. By applying the map $T$ on the regions above and below the curve $y=x$, one can observe that a nonequilibrium solution of (3) must be oscillatory. Also, using the map $T$, one can observe that stocking increases the frequency of oscillations in the following sense. The length of semicycles in the absence of stocking is longer than the length of semicycles in the existence of stocking, where a semicycle is used to denote the string of consecutive terms above or below the equilibrium.

Since solutions of (3) are bounded, we define

$$
S:=\lim \sup x_{n}, \quad I:=\lim \inf x_{n} .
$$

From the equation $x_{n+2}=x_{n} f\left(x_{n}\right) f\left(x_{n-1}\right)+h f\left(x_{n}\right)+h$ and using the fact that $t f(t)$ is increasing, we obtain

$$
\begin{aligned}
& S \leq S f(S) f(I)+h f(I)+h, \\
& I \geq I f(I) f(S)+h f(S)+h .
\end{aligned}
$$

When $h>0$, we have $S \geq I>0$. So, we can multiply the first inequality by $I$ and the second one by $S$ to obtain

$$
S(f(S)+1) \leq I(f(I)+1) .
$$

Since $t(f(t)+1)$ is increasing, we obtain $I=S$. This approach was used by Camouzis and Ladas in [22], and it was used by Nyerges in [21] to prove that $\bar{x}_{2, h}$ is globally attractive. This fact together with the local stability established in Proposition 1 shows the global asymptotic stability of $\bar{x}_{2, h}$ as we summarize in the following proposition.

Proposition 3. The equilibrium solution $\bar{x}_{2, h}$ of (3) is globally asymptotically stable.

Next, it is obvious that the positive quadrant forms an invariant for (3); however, since solutions are bounded, we are interested in a bounded invariant that can be developed to serve us in the periodic case. Notice that by invariance here we always mean forward invariance, that is, $R_{h}$ is an invariant of (3) if $T(x, y) \in R_{h}$ for all $(x, y) \in R_{h}$. To establish the existence of a bounded invariant region, we need to have in mind the following simple fact.

Proposition 4. There exists a finite constant $c_{h} \geq h$ such that $G_{h}(t)=(b t+h) f(t) \leq b c_{h}$ for all $t \geq 0$. Furthermore, $c_{h}$ can be taken as $c_{h}:=(1 / b) \sup _{t} G_{h}(t)$.

Proof. Use the fact that $t f(t)$ is bounded and $f(t)$ is decreasing with $f(0)=b$ and $\lim _{t \rightarrow \infty} f(t)=0$ to obtain the result.

Next, define the curves $\Gamma_{j}, j=0,1,2,3,4$ to be the line segments that connect the points $(0,0),(0, h),\left(c_{h}, b c_{h}+h\right)$, $\left(b c_{h}+h, b c_{h}+h\right),\left(b c_{h}+h, 0\right)$, and $(0,0)$, respectively. Now, define $R_{h}$ to be the region bounded by the curves of $\Gamma_{j}, j=0, \ldots, 4$ including the boundary, then the following result gives a bounded invariant of (3). Here, it is worth mentioning that $\Gamma_{0}$ shrinks to a point at $h=0$; however, our notation and arguments about the invariant region are still valid except that the boundary of $R_{h}$ becomes a quadrilateral rather than a pentagon.

Theorem 5. The region $R_{h}$ as defined above gives a compact invariant for (3).

Proof. Consider the map $T(x, y)$ as defined in (7). $T$ is one-toone on the positive quadrant. Thus, all we need is to test $T$ on the boundary of $R_{h}$. It is straightforward computations to find that $T\left(\Gamma_{0}\right) \subseteq \Gamma_{1}$. Since horizontal line segments are mapped to vertical line segments under $T$, we test the end points of $\Gamma_{2}$ to find

$$
\begin{gathered}
T\left(c_{h}, b c_{h}+h\right)=\left(b c_{h}+h,\left(b c_{h}+h\right) f\left(c_{h}\right)+h\right), \\
T\left(b c_{h}+h, b c_{h}+h\right) \\
=\left(b c_{h}+h,\left(b c_{h}+h\right) f\left(b c_{h}+h\right)+h\right) .
\end{gathered}
$$

By the choice of $c_{h}$ given in Proposition 4 , we have

$$
\begin{aligned}
& \left(b c_{h}+h\right) f\left(b c_{h}+h\right)+h \\
& \quad \leq\left(b c_{h}+h\right) f\left(c_{h}\right)+h \leq b c_{h}+h .
\end{aligned}
$$


Thus, $T\left(\Gamma_{2}\right) \subset \Gamma_{3}$. Next, $T\left(\Gamma_{3}\right) \subset R_{h}$ and $T\left(\Gamma_{4}\right)=(0, h)$ are straightforward to observe. Finally, we show that $T\left(\Gamma_{1}\right) \subset R_{h}$. For $0 \leq t \leq c_{h}$, we have

$$
T(t, b t+h)=(b t+h,(b t+h) f(t)+h) ;
$$

however, $h \leq b t+h \leq b c_{h}+h$ and $(b t+h) f(t)+h \leq b c_{h}+h$ by the choice of $c_{h}$, which completes the proof. Figure 2 illustrates the region $R_{h}$ and its image under the map $T$ when $(b t+h) f(t)$ is increasing.

\section{Periodic Stocking}

In this section, we force periodic stocking on (3) to obtain

$$
x_{n+1}=x_{n} f\left(x_{n-1}\right)+h_{n},
$$

where $h_{n}$ is a $p$-periodic sequence of stocking quotas, and $p$ denotes the minimal period. Observe that some consecutive values of the stocking sequence can be zero; however, it is natural to assume that $\sum_{j=0}^{p-1} h_{j}>0$. As in the constant case, we associate (14) with a $p$-periodic sequence of two dimensional maps that we use in the sequel, namely $\left\{T_{j}, j=0,1, \ldots, p-1\right\}$, where $T_{j}(x, y)=\left(y, y f(x)+h_{j}\right)$. It is obvious that if we replace $h$ by $h_{j}$ in Theorem 5 , then $R_{h_{j}}$ forms a compact and invariant region for the individual map $T_{j}$, which enables us to build a suitable machinery for establishing the existence of a periodic solution. It is convenient now to develop the notations of the previous section so it can suit the periodic case. We denote the line segments that form the boundary of $R_{h_{j}}$ by $\Gamma_{j, i}, i=0, \ldots, 4$, where $\Gamma_{j, i}$ corresponds to $\Gamma_{i}$ in the autonomous case and that are associated with the individual $\operatorname{map} T_{j}$. Also, the constant $c_{h}$ in Proposition 4 will be replaced by $c_{h_{j}}$, and this is associated with the individual map $T_{j}$.

3.1. Existence of a Periodic Solution. We start by establishing a compact invariant region for (14). Define

$$
\begin{gathered}
h_{m}:=\max \left\{h_{0}, h_{1}, \ldots, h_{p-1}\right\}, \\
c_{m}:=\max \left\{c_{h_{j}}: j=0, \ldots, p-1\right\},
\end{gathered}
$$

where $c_{h_{j}}$ is as taken in Proposition 4; that is, $c_{h_{j}}=$ $(1 / b) \sup _{t} G_{h_{j}}(t)$; then use $h_{m}$ and $c_{m}$ to define the region $R_{h_{m}}$ as in the paragraph preceding Theorem 5. Now, we have the following result.

Lemma 6. Consider (14) together with the associated pperiodic sequence of maps $\left\{T_{j}\right\}$. Each of the following holds true.

(i) One has $R_{h_{i}} \subseteq R_{h_{j}}$ whenever $h_{i} \leq h_{j}$.

(ii) $R_{h_{m}}$ is a compact invariant for each individual map $T_{j}$.

(iii) $R_{h_{m}}$ is a compact invariant for the map $\widehat{T}:=T_{p-1}$ 。 $T_{p-2} \circ \cdots \circ T_{0}$.

Proof. (i) When $h_{i} \leq h_{j}$, we obtain $G_{h_{i}}(t) \leq G_{h_{j}}(t)$ for all $t \geq 0$. Thus, $c_{h_{i}} \leq c_{h_{j}}$, and the result becomes obvious from
Proposition 4 and the geometric structure of the regions $R_{h_{i}}$ and $R_{h_{j}}$. To prove (ii), let $(x, y) \in R_{h_{m}}$, we show that $T_{j}(x, y) \in$ $R_{h_{m}}$. Since

$$
\begin{aligned}
T_{j}(x, y) & =\left(y, y f(x)+h_{j}\right) \\
& =\left(y, y f(x)+h_{m}\right)-\left(0, h_{m}-h_{j}\right) \\
& =T_{m}(x, y)-\left(0, h_{m}-h_{j}\right),
\end{aligned}
$$

then the first component of $T_{j}(x, y)$ is the same as the first component of $T_{m}(x, y)$ and the second component of $T_{j}(x, y)$ is lower than the second component of $T_{m}(x, y)$. Now, the fact that $T_{m}(x, y) \in R_{h_{m}}$ and the geometric structure of $R_{h_{m}}$ assures that $T_{j}(x, y) \in R_{h_{m}}$. Finally, (iii) follows from (ii).

Periodic stocking (or harvesting) has the effect of forcing population cycles to evolve and become multiples of the stocking/harvesting period as we show in the following result, which is more general than (14).

Theorem 7. Consider the general difference equation $x_{n+1}=$ $F\left(x_{n}, x_{n-1}, \ldots, x_{n-k}\right)$ with p-periodic stocking (or harvesting). If a periodic solution exists, then the period is a multiple of $p$.

Proof. The proof is a contradiction; suppose that we have an $r$-periodic solution of the equation $x_{n+1}=F\left(x_{n}, x_{n-1}, \ldots\right.$, $\left.x_{n-k}\right)+h_{n}$ for some $r$ that is not a multiple of $p$. Then, the greatest common divisor between $r$ and $p(d:=\operatorname{gcd}(r, p))$ is not $p$. Define the maps $F_{i}:=F+h_{i}, i=0,1, \ldots, p-1$; then for each $0 \leq i \leq d-1$, the maps $\left\{F_{k d+i}, k=0,1, \ldots, p / d-1\right\}$ must agree at the point $X_{i}:=\left(x_{i}, x_{i-1}, \ldots, x_{i-k}\right)$, where the components $x_{i-k}, x_{i+1-k}, \ldots, x_{i}$ are consecutive elements of the $p$-periodic solution. This implies

$$
h_{i}=h_{d+i}=h_{2 d+i}=\cdots=h_{(p / d-1) d+i}
$$

for all $i=0,1, \ldots, d-1$, which contradicts the minimality of the period of the $p$-periodic difference equation.

Theorem 7 shows that (14) has no equilibrium solutions, and therefore, our previous notion of characterizing oscillatory solutions based on the oscillations about $y=x$ is the valid one here. Thus, solutions of (14) are oscillatory about $y=x$ because they cannot be monotonic. Although it is natural for fluctuations in the environment to create fluctuations in the population, we find it appropriate here to connect the loosely-defined term "fluctuation" with the mathematically well-defined term "oscillation." Next, we use the Brouwer fixed theorem [23] (page 51) to prove the existence of a periodic solution of (14).

Lemma 8 (Brouwer fixed-point theorem [23], page 51). Let $M$ be a nonempty, convex, and compact subset of $\mathbb{R}^{n}$. If $T$ : $M \rightarrow M$ is continuous, then $T$ has a fixed point in $M$.

Theorem 9. The p-periodic difference equation in (14) has a p-periodic solution. 

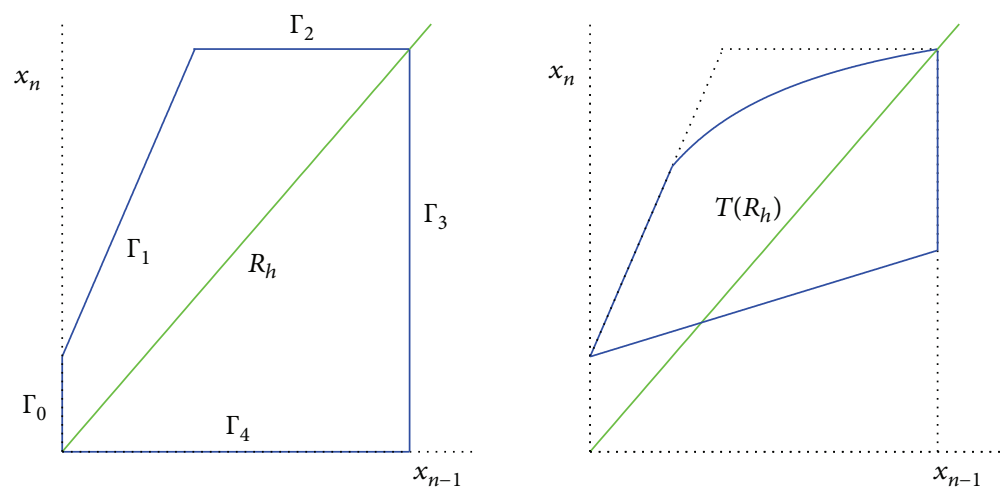

Figure 2: The figure on the left shows the choice of the compact region $R_{h}$ when $y=(b t+h) f(t)$ is increasing, and the one on the right shows $T\left(R_{h}\right)$ with blue boundary inside $R_{h}$.

Proof. Consider the map $\widehat{T}:=T_{p-1} \circ T_{p-2} \circ \cdots \circ T_{0}$, then using Lemma 6, we obtain $\widehat{T}: R_{h_{m}} \rightarrow R_{h_{m}}$. Furthermore, $R_{h_{m}}$ is nonempty, compact, and obviously convex. So, by Lemma 8 , $\widehat{T}$ has a fixed point in $R_{h_{m}}$. This fixed point establishes a periodic solution of (14) with minimal period that divides $p$; however, Theorem 7 shows that the period must be $p$.

3.2. Global Attractivity of the Periodic Solution When $p=2$. Consider the periodicity of (14) to be $p=2$ and suppose $h_{0}+h_{1} \neq 0$. We partition the solutions of (14) into two subsequences, the one with even indices $\left\{x_{2 n}\right\}$ and the one with odd indices $\left\{x_{2 n}\right\}$. Thus, we have

$$
\begin{aligned}
& x_{2 n+1}=x_{2 n} f\left(x_{2 n-1}\right)+h_{0}, \\
& x_{2 n+2}=x_{2 n+1} f\left(x_{2 n}\right)+h_{1} .
\end{aligned}
$$

Since the solutions are bounded, we define

$\liminf \left\{x_{2 n+i}\right\}=I_{i}, \quad \limsup \left\{x_{2 n+i}\right\}=S_{i}, \quad i=0,1$.

Now, the second iterate of (18) gives us

$$
\begin{gathered}
x_{2 n+2}=x_{2 n} f\left(x_{2 n}\right) f\left(x_{2 n-1}\right)+h_{0} f\left(x_{2 n}\right)+h_{1}, \\
x_{2 n+3}=x_{2 n+1} f\left(x_{2 n+1}\right) f\left(x_{2 n}\right)+h_{1} f\left(x_{2 n+1}\right)+h_{0} .
\end{gathered}
$$

Use the fact that $f(t)$ is decreasing and $t f(t)$ is increasing in (20) to obtain

$$
\begin{aligned}
& S_{0} \leq S_{0} f\left(S_{0}\right) f\left(I_{1}\right)+h_{0} f\left(I_{0}\right)+h_{1}, \\
& I_{0} \geq I_{0} f\left(I_{0}\right) f\left(S_{1}\right)+h_{0} f\left(S_{0}\right)+h_{1} .
\end{aligned}
$$

Also, (21) gives us

$$
\begin{aligned}
& S_{1} \leq S_{1} f\left(S_{1}\right) f\left(I_{0}\right)+h_{1} f\left(I_{1}\right)+h_{0}, \\
& I_{1} \geq I_{1} f\left(I_{1}\right) f\left(S_{0}\right)+h_{1} f\left(S_{1}\right)+h_{0} .
\end{aligned}
$$

Multiply inequality (22) by $I_{0}$ and inequality (23) by $S_{0}$ to obtain

$$
\begin{aligned}
S_{0} I_{0} f & \left(I_{0}\right) f\left(S_{1}\right)+S_{0}\left(h_{0} f\left(S_{0}\right)+h_{1}\right) \\
& \leq I_{0} S_{0} f\left(S_{0}\right) f\left(I_{1}\right)+I_{0}\left(h_{0} f\left(I_{0}\right)+h_{1}\right) .
\end{aligned}
$$

Since $I_{0}\left(h_{0} f\left(I_{0}\right)+h_{1}\right) \leq S_{0}\left(h_{0} f\left(S_{0}\right)+h_{1}\right)$, we obtain

$$
f\left(I_{0}\right) f\left(S_{1}\right) \leq f\left(S_{0}\right) f\left(I_{1}\right) .
$$

Also, multiply inequality (24) by $I_{1}$ and inequality (25) by $S_{1}$ to obtain

$$
\begin{aligned}
& S_{1} I_{1} f\left(I_{1}\right) f\left(S_{0}\right)+S_{1}\left(h_{1} f\left(S_{1}\right)+h_{0}\right) \\
& \quad \leq I_{1} S_{1} f\left(S_{1}\right) f\left(I_{0}\right)+I_{1}\left(h_{1} f\left(I_{1}\right)+h_{0}\right) .
\end{aligned}
$$

Since $I_{1}\left(h_{1} f\left(I_{1}\right)+h_{0}\right) \leq S_{1}\left(h_{1} f\left(S_{1}\right)+h_{0}\right)$, we obtain

$$
f\left(I_{1}\right) f\left(S_{0}\right) \leq f\left(I_{0}\right) f\left(S_{1}\right) .
$$
result.

Using inequalities (27) and (29), we obtain the following

Lemma 10. Consider $I_{0}, I_{1}, S_{0}, S_{1}$ as defined in (19); then $f\left(I_{0}\right) f\left(S_{1}\right)=f\left(I_{1}\right) f\left(S_{0}\right)$.

Next, we give the following result.

Theorem 11. For $p=2$, the 2-periodic solution of (14) is a global attractor.

Proof. Use the result of Lemma 10 in inequality (26) to obtain

$$
S_{0}\left(h_{0} f\left(S_{0}\right)+h_{1}\right) \leq I_{0}\left(h_{0} f\left(I_{0}\right)+h_{1}\right) .
$$

Since $g(t)=t\left(h_{0} f(t)+h_{1}\right)$ is increasing and $I_{0} \leq S_{0}$, we must have $I_{0}=S_{0}$. Similarly, use the result of Lemma 10 in inequality (28) to obtain

$$
S_{1}\left(h_{1} f\left(S_{1}\right)+h_{0}\right) \leq I_{1}\left(h_{1} f\left(I_{1}\right)+h_{0}\right),
$$

and consequently $S_{1}=I_{1}$. Hence, $I_{i}=S_{i}, i=0,1$, and the proof is complete.

Remark 12. Observe that the approach of this section proves not only the global attractivity of the $p$-periodic solution but also its existence; however, Theorem 7 is still significant here because it proves the minimality of the period. Also, establishing the compact invariant region in Lemma 6 deserves embracing regardless of the global attractivity of the periodic solution. Finally, proving the global attractivity for general $p$ will be the topic of some future work. 


\section{Pielou's Equation with Stocking}

As an illustrative example to our results, we consider the function $f$ in (14) to be $f(t)=b t /(1+t)$. It is worth mentioning that in the absence of stocking, Pielou ([24], page 80) suggested taking $f\left(x_{n-m}\right)=\mu K /\left(K+(\mu-1) x_{n-m}\right)$ to account for certain fluctuating populations, which cannot be modeled by the Beverton-Holt equation. So, here we are dealing with the dimensionless Pielou's equation $y_{n+1}=$ $b y_{n} /\left(1+y_{n-1}\right)$, which takes the following form after forcing stocking:

$$
y_{n+1}=\frac{b y_{n}}{1+y_{n-1}}+h_{n}
$$

When $h_{n}=0$, (32) has the positive equilibrium $x_{2,0}=$ $b-1$ which is globally asymptotically stable. When $h_{n}=h>$ $0, x_{2, h}=(1 / 2)(b+h-1)+(1 / 2) \sqrt{(b+h-1)^{2}+h}$ inherits the global asymptotic stability of $x_{2,0}$ as shown in [21]. Now, consider $\left\{h_{n}\right\}$ to be 2 -periodic. To find the 2-periodic solution assured by Theorem 9 , we substitute $y_{-1}=x$ and $y_{0}=y$ in (32) to obtain

$$
\left(y-h_{0}\right)(1+y)=b x, \quad\left(x-h_{1}\right)(1+x)=b y .
$$

Now, the solution is obvious graphically (it is the point of intersection between the two curves in the positive quadrant). However, the solution is not simple to write explicitly, and therefore, we proceed by choosing $h_{0}=b+1 /(b-1)$ and $h_{1}=b$. In this case, the 2 -periodic solution $\{\bar{x}, \bar{y}\}$ is given by

$$
\bar{x}=b-1+\frac{b^{3 / 2}}{\sqrt{b-1}}, \quad \bar{y}=b+\frac{1}{b-1}+\sqrt{b(b-1)} .
$$

Next, use the function $f(t)=b /(1+t)$ in Section 3.2 and follow the same steps to find

$$
\begin{gathered}
\liminf \left\{y_{2 n}\right\}=I_{0}=S_{0}=\lim \sup \left\{y_{2 n}\right\}, \\
\liminf \left\{y_{2 n+1}\right\}=I_{1}=S_{1}=\lim \sup \left\{y_{2 n+1}\right\} .
\end{gathered}
$$

Thus, the odd iterates converge to a point, say $u$, while the even iterates converge to a point, say $v$. Substitute $u$ and $v$ in (18); then compare it with (33) to find that $\{u, v\}$ is indeed $\{\bar{x}, \bar{y}\}$. Finally, Figure 3 shows the convergence to the 2-cycle for the specific values of the parameters.

Another interesting notion that can be observed here is the resonance of the solutions of (32). The arithmetic average of the globally attracting 2-periodic solution is

$$
\begin{aligned}
x_{s v} & :=\frac{1}{2}(\bar{x}+\bar{y}) \\
& =\frac{1}{2}\left(2 b-1+\frac{1}{b-1}+\frac{b^{3 / 2}}{\sqrt{b-1}}+\sqrt{b(b-1)}\right) .
\end{aligned}
$$

On the other hand, when we take the constant stocking $h=(1 / 2)\left(h_{0}+h_{1}\right)=b+1 / 2(b-1)$, we obtain the globally attracting equilibrium

$$
\bar{x}=\frac{4 b^{2}-6 b+3+\sqrt{16 b^{4}-32 b^{3}+28 b^{2}-12 b+1}}{4(b-1)} .
$$

Figure 4 shows that $x_{a v}>\bar{x}$.

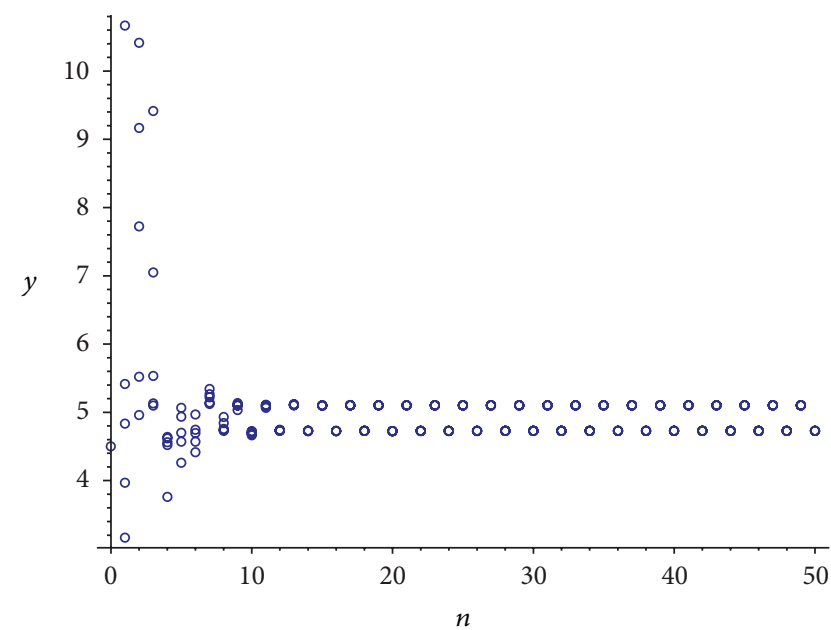

FIgURE 3: This graph shows the stable 2-cycle for the 2-periodic equation in (32) when $h_{0}=b+1 /(b-1)$ and $h_{1}=b$, where $b$ is fixed at $5 / 2$.

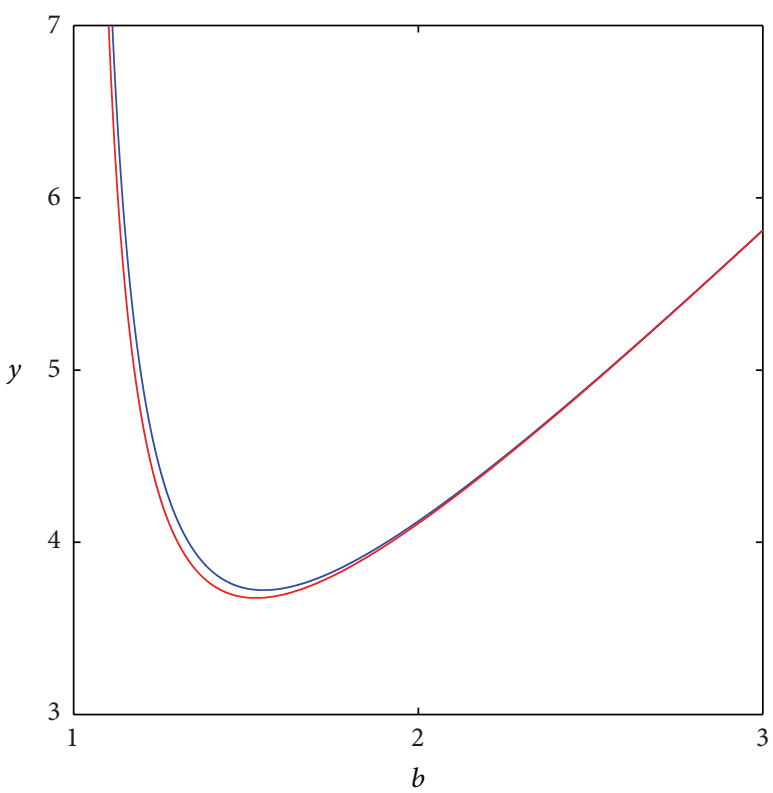

Figure 4: This graph shows the average of the attracting 2-cycle (blue color) in contrast with the equilibrium that results from constant stoking equals the average of $h_{0}$ and $h_{1}$, where $h_{0}=b+$ $1 /(b-1)$ and $h_{1}=b$.

\section{Conclusion and Discussion}

In this paper, we investigated the dynamics of the periodic difference equation $x_{n+1}=x_{n} f\left(x_{n-1}\right)+h_{n}$, where $f(x)$ is differentiable and decreasing on $[0, \infty)$, while $x f(x)$ is increasing and bounded. This equation can be used as a discrete model to represent contest competition in species with periodic stocking. We found that periodic stocking forces the existence of a periodic solution that has the same period as the stocking period. In addition to the unbounded invariant given by the positive quadrant, we constructed 
a bounded invariant region, which we used to prove the existence of the periodic solution. Also, we proved that the periodic solution is globally attractive when the stocking period is 2 . We conjecture that the periodic solution is globally attractive regardless of the stocking period. Although the steady state has evolved to become the periodic solution of the same period as the stocking period, our results show that periodic stocking preserves the global attractivity of the periodic solution.

\section{Acknowledgments}

The author thanks one of the anonymous referees for his suggestions and remarks. This work is partially supported by SQU Internal Grant IG/SCI/DOMS/11/14.

\section{References}

[1] M. P. Hassell, "Density-dependence in single-species populations," The Journal of Animal Ecology, vol. 44, pp. 283-295, 1975.

[2] M. Kot, Elements of Mathematical Ecology, Cambridge University Press, Cambridge, Uk, 2001.

[3] Å. Brännström and D. J. T. Sumpter, "The role of competition and clustering in population dynamics," Proceedings of the Royal Society B, vol. 272, no. 1576, pp. 2065-2072, 2005.

[4] G. C. Varley, G. R. Gradwell, and M. P. Hassell, Insect Population Ecology, Blackwell Scientific, Oxford, UK, 1973.

[5] S. M. Henson and J. M. Gushing, "Hierarchical models of intra-specific competition: scramble versus contest," Journal of Mathematical Biology, vol. 34, no. 7, pp. 755-772, 1996.

[6] R. Beverton and S. J. Holt, On the Dynamics of Exploited Fish Populations, The Blackburn Press, New Jersey, NJ, USA, 2004.

[7] S. A. Levin and R. M. May, "A note on difference-delay equations," Theoretical Population Biology, vol. 9, no. 2, pp. 178187, 1976.

[8] R. M. May, "Time-delay versus stability in population models with two and three trophic levels," Ecology, vol. 54, pp. 315-325, 1973.

[9] L. Nunney, "Short time delays in population models: a role in enhancing stability," Ecology, vol. 66, no. 6, pp. 1849-1858, 1985.

[10] C. E. Taylor and R. R. Sokal, "Oscillations in housefly population size due to time lags," Ecology, vol. 57, pp. 1060-1067, 1976.

[11] V. L. Kocić and G. Ladas, Global Behavior of Nonlinear Difference Equations of Higher Order with Applications, vol. 256, Kluwer Academic, Dordrecht, The Netherlands, 1993.

[12] H. I. McCallum, "Effects of immigration on chaotic population dynamics," Journal of Theoretical Biology, vol. 154, no. 3, pp. 277284, 1992.

[13] G. D. Ruxton, "Low levels of immigration between chaotic populations can reduce system extinctions by inducing asynchronous regular cycles," Proceedings of the Royal Society B, vol. 256, no. 1346, pp. 189-193, 1994.

[14] G. D. Ruxton, "The effect of emigration and immigration on the dynamics of a discrete-generation population," Journal of Biosciences, vol. 20, no. 3, pp. 397-407, 1995.

[15] G. D. Ruxton and P. Rohani, "Population floors and the persistence of chaos in ecological models," Theoretical Population Biology, vol. 53, no. 3, pp. 175-183, 1998.
[16] S. Sinha and P. K. Das, "Dynamics of simple one-dimensional maps under perturbation," Pramana, vol. 48, no. 1, pp. 87-98, 1997.

[17] L. Stone, "Period-doubling reversals and chaos in simple ecological models," Nature, vol. 365, no. 6447, pp. 617-620, 1993.

[18] L. Stone and D. Hart, "Effects of immigration on the dynamics of simple population models," Theoretical Population Biology, vol. 55, no. 3, pp. 227-234, 1999.

[19] P. Sun and X. B. Yang, "Dynamic behaviors of the Ricker population model under a set of randomized perturbations," Mathematical Biosciences, vol. 164, no. 2, pp. 147-159, 2000.

[20] R. Abu-Saris, Z. AlSharawi, and M. Rhouma, "The dynamics of some discrete models with delay under the effect of constant yield harvesting," Chaos, Solitons \& Fractals, vol. 54, pp. 26-38, 2013.

[21] G. Nyerges, "A note on a generalization of Pielou's equation," Journal of Difference Equations and Applications, vol. 14, no. 5, pp. 563-565, 2008.

[22] E. Camouzis and G. Ladas, "Periodically forced Pielou's equation," Journal of Mathematical Analysis and Applications, vol. 333, no. 1, pp. 117-127, 2007.

[23] E. Zeidler, Nonlinear Functional Analysis and Its Applications. I, Springer, New York, NY, USA, 1986.

[24] E. C. Pielou, Population and Community Ecology, Gordon and Breach, New York, NY, USA, 1974. 


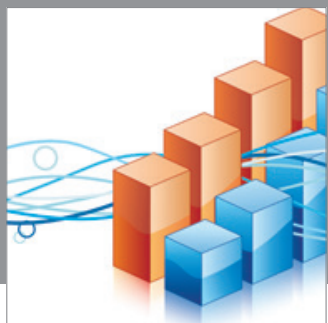

Advances in

Operations Research

mansans

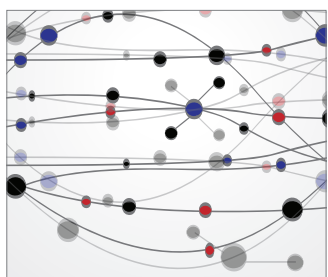

The Scientific World Journal
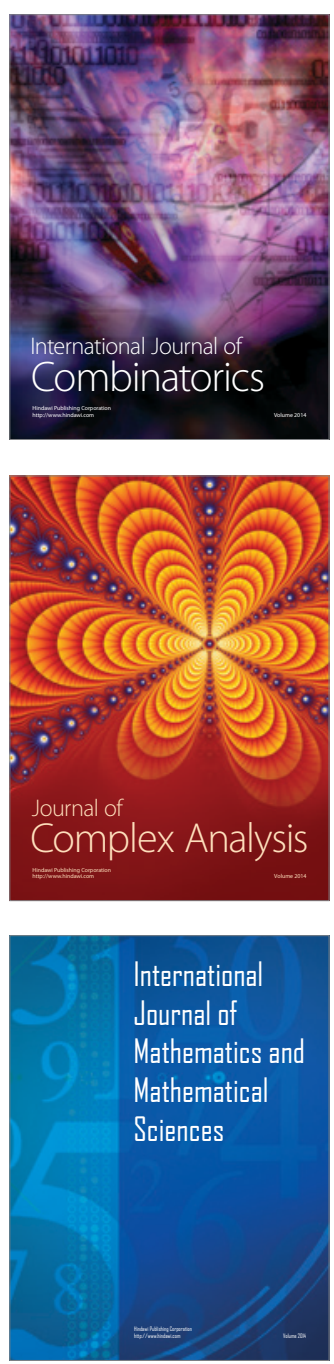
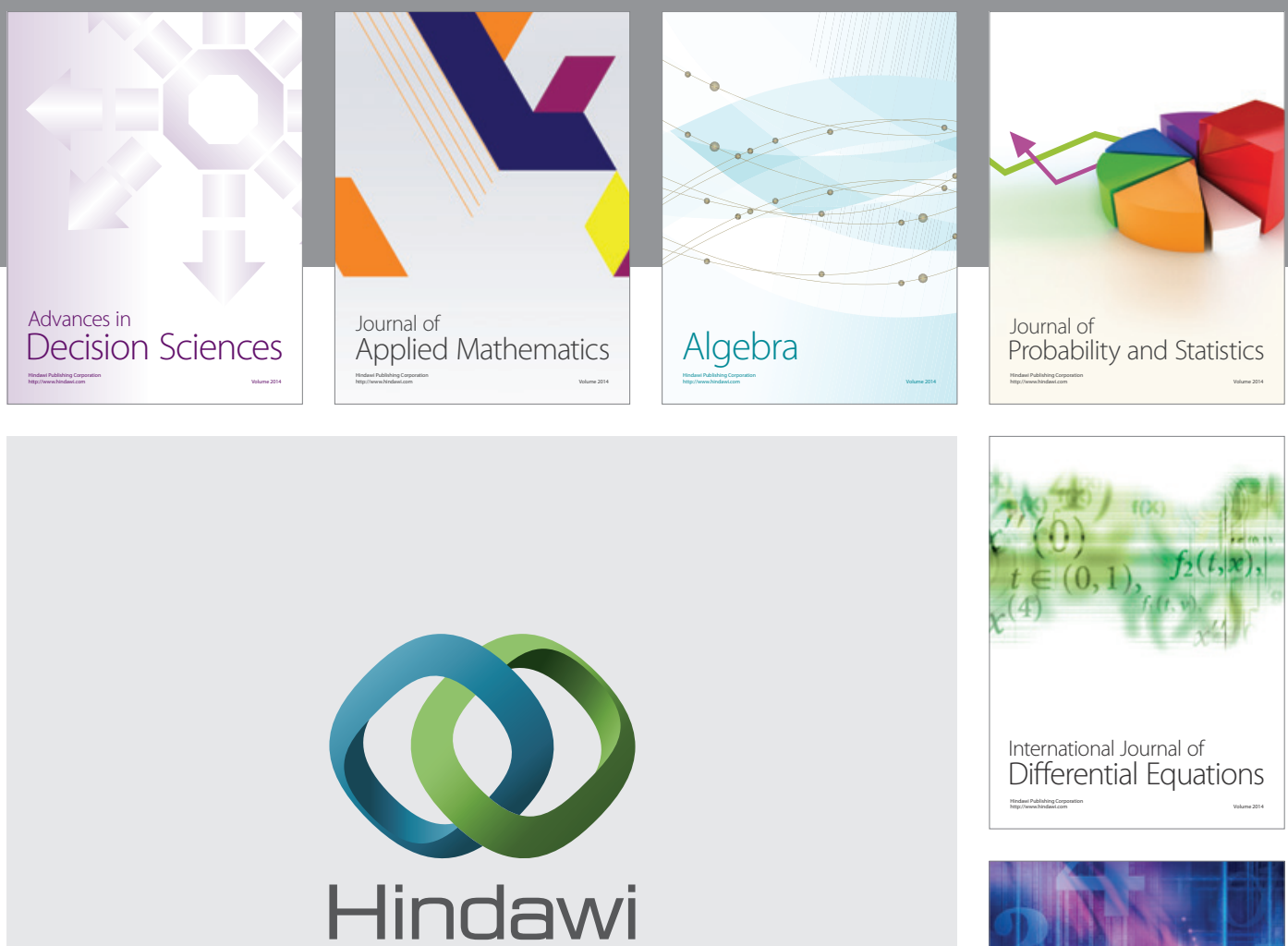

Submit your manuscripts at http://www.hindawi.com
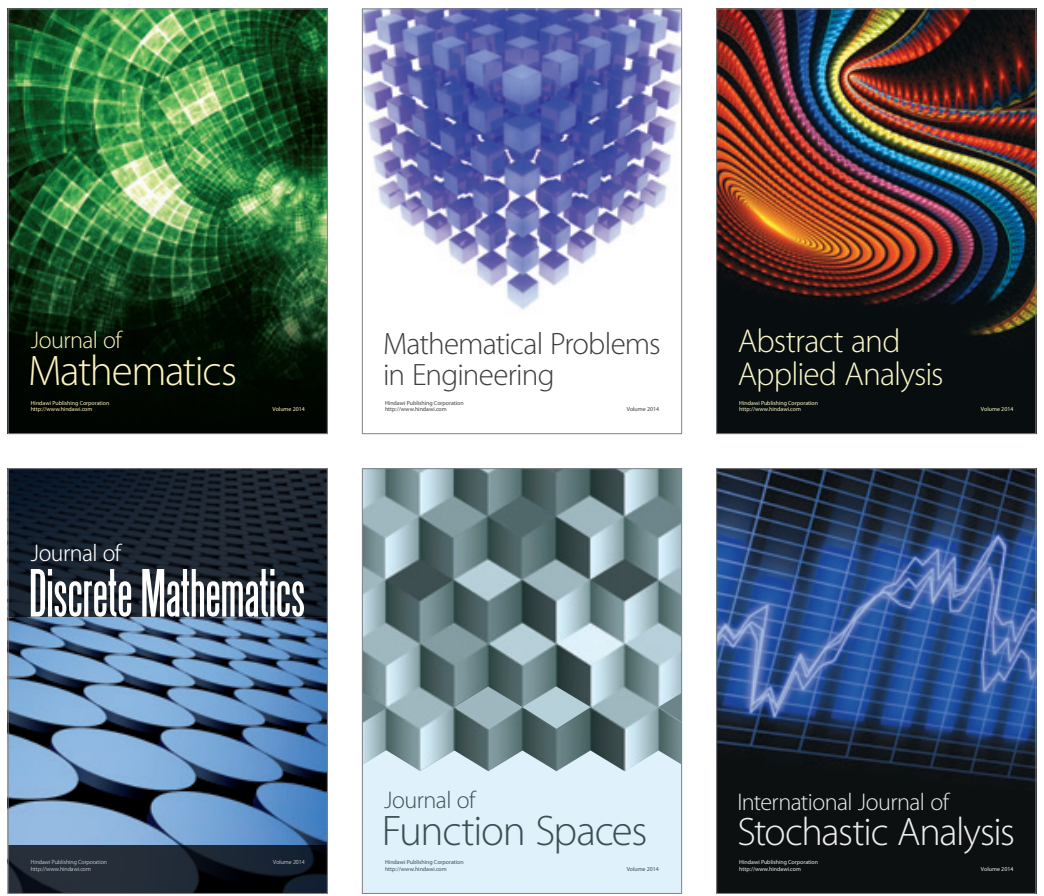

Journal of

Function Spaces

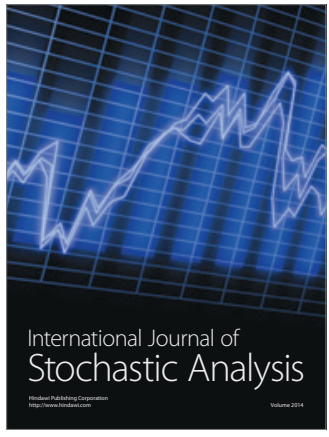

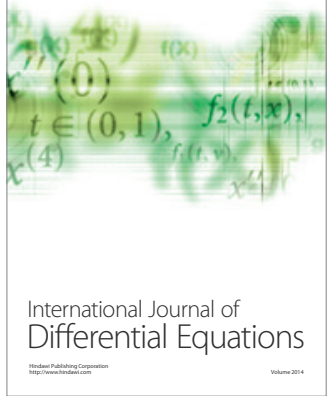
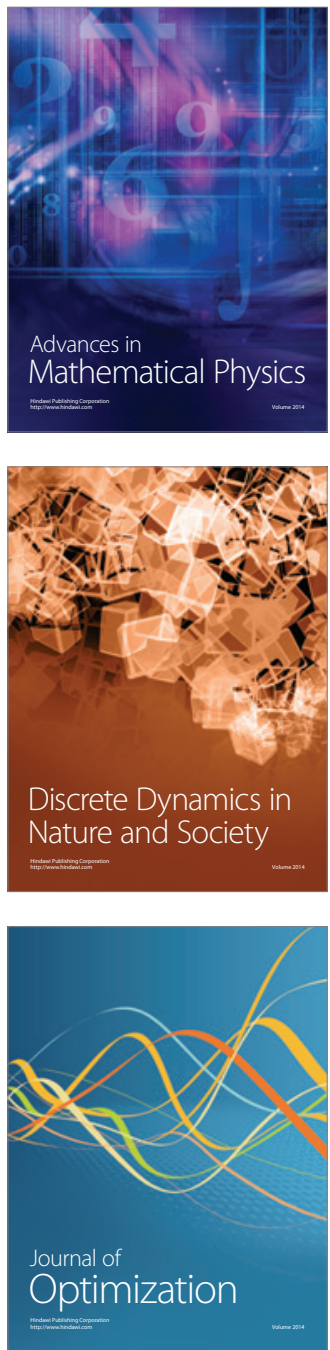